\title{
O impacto da saúde bucal na qualidade de vida de crianças infectadas pelo HIV: revisão de literatura
}

\author{
The impact of oral health on the quality of life \\ of HIV infected children: a literature review
}

Ana Karla Buczynski ${ }^{1}$

Glória Fernanda Castro ${ }^{1}$

I vete Pomarico Ribeiro de Souza ${ }^{1}$

\footnotetext{
${ }^{1}$ Departamento de Odontopediatriae Ortodontia, Faculdadede Odontologia, Universidade Federal do Rio de Janeiro. Av. Brigadeiro Trompowsky $\mathrm{s} / \mathrm{n}$, Cidade U niversitária. 21941-590. Rio de Janeiro RJ.anabodonto@yahoo.com
}

Abstract The search for improvement of the health of systemically compromised patients and for a better knowledge about the impact of diseases on their lives has brought great interest for health-related quality of life, mainly in children with chronic diseases. The quality of life related to oral health is thus relevant, not only for being an inseparable component of the general health but also due to the importance of oral problems in the lives of these patients. The evaluation of oral health-related quality of life in HIV infected children can be of great importance seen that these patients show high prevalence of caries and periodontal diseases besides the oral manifestations of the virus infection itself. The aim of this article is to present some concepts about quality of life and the use of instruments for its evaluation on the basis of a literature review as well as to analyze the impact of oral health on the quality of life of HIV infected children.

Key words Child, HIV infections, Quality of life, Oral health
Resumo Em busca de uma melhora da saúde de pacientes sistemicamente comprometidos e um maior entendimento do impacto de doenças nas suas vidas, grande interesse tem sido dado à qualidadedevida relacionada à saúde, principalmente em crianças com doenças crônicas. N este sentido, a qualidade de vida relacionada à saúde bucal tem sua importância haja vista que a mesma é um componente indissociável da saúde geral e também pela relevância dos problemas orais na vida destes pacientes. Assim, a avaliação de qualidade de vida relacionada à saúde bucal em pacientes infantis infectados pelo HIV pode ser de grande relevância uma vez queestasapresentam alta prevalência de doença cárie e periodontal, além da presença de manifestações orais da própria infecção pelo vírus. Dessa forma, o objetivo deste artigo é, através de uma revisão de literatura, apresentar alguns conceitos relacionados à qualidade de vida e utilização de instrumentos de avaliação da mesma, bem como analisar o impacto da saúde bucal na qualidade de vida de crianças infectadas pelo HIV.

Palavras-chave Criança, Infecções por HIV, Qualidade devida, Saúde bucal 
Introdução

A saúde bucal (SB) é um componente indissociável da saúde geral, por isso tem havido grande interesse pelo desenvolvimento e utilização de instrumentos de mensuração de qualidade de vida relacionado à saúde (QVRS), com o objetivo de avaliar o impacto da doença na vida do indivíduo. Em crianças, a QVRS deve ser considerada de forma diferenciada de adultos ${ }^{1}$. A avaliação da qualidade de vida (QV) em populações infantis com doença crônica permite determinar de maneira significativa o impacto dos cuidados com a saúde quando a cura não é possível2.

Dentre as doenças crônicas de grande relevância para os pacientes infantis, destaca-se a infecção pelo HIV, uma vez que tiveram sua sobrevida aumentada com a terapia anti-retroviral, o que proporciona uma melhora significativa da QV para estes pacientes. Muitos dos que nasceram infectados na década de 1980, hoje, estão com a doença sob controle, tornaram-se adultos e estão tendo filhos sem 0 vírus, graças aos avanços da medicina. No entanto, ainda é significativo o número de crianças que convivem com os sintomas da doença, tornando-se urgente e essencial a busca de instrumentos que sejam capazes de relacionar a QV com a saúdee, ainda, o impacto da SB na vida dessas crianças, uma vez que a prevalência de manifestações orais ou de problemas gerados pela doença ainda são muito altos ${ }^{3,4}$.

Cabe destacar que os indicadores tradicionais (morbidade, CPO-D, índice de placa, entre outros) são capazes de avaliar a patologia, mas são limitados quando se pretende avaliar os efeitos da doença ${ }^{5}$, principalmente no caso do indivíduo em desenvolvimento. Assim, merecido destaque tem sido dado a avaliação não só da presença da enfermidade causada pelo vírus HIV, como também da qualidade de vida destes pacientes infantis, em virtude deste ser um componente auxiliar na mensuração da saúde do paciente. Dentro deste contexto, objetiva-se, através de uma revisão de literatura, apresentar alguns conceitos relacionados à qualidade de vida e à utilização de instrumentos de avaliação da mesma, eainda analisar o impacto da saúde bucal na qualidadedevida de crianças infectadas pelo HIV.

\section{Qualidade de vida e saúde bucal}

O Grupo de Qualidade de Vida da Organização M undial de Saúde 6 definiu QV como "a percep- ção do indivíduo de sua posição na vida, no contexto da cultura esistema devalores nos quais ele viveeem relação aos seus objetivos, expectativas, padrões e preocupações". Nesta definição, fica implícito queo conceito de QV ésubjetivo, multidimensional equeinclui elementos de avaliação tanto positivos quanto negativos?

Basto ${ }^{8}$ destaca que, uma vez quea QV decorre dos aspectos sociais, econômicos, políticos e culturais de uma sociedade, a problemática das doenças que afetam os indivíduos já não pode mais ser explicada unicamente pel os fatores biológicos que as caracterizam. Classificar a saúde em boa, má ou razoável é também definir a QV, pois ela surge das condiç̧̃̃es da classe social, das relações no trabalho, da alimentação, da moradia, do saneamento básico, do meio ambientesaudável, do acesso à educação, ao transporte, ao lazer, aos serviços de saúde, enfim, detudo o que diz respeito à vida. D essa forma, como as doenças refletem de várias formas na vida do indivíduo, a conceituação do termo saúde adquire uma complexidade muito grande, tendo em vista os vários aspectos que envolvem a vida em sociedade.

Em 1948, a Organização Mundial de Saúde (OMS) definiu saúde como "o completo bemestar físico, mental e social, e não meramente a ausência de doença". Esse éum conceito biológico, pertencente ao paradigma médico, com seu foco em agentes etiológicos eem resultados clínicos, cujas origens filosóficas do modelo são encontradas no dualismo do corpo-mente no qual a mente e o corpo são entidades separadas, e a saúde e a doença são vistas como fenômenos estritamente biológicos. A alta tecnologia e os serviços de saúde são vistos como sendo uma chave para restaurar e melhorar a saúde tanto individual quanto de populações. Como resultados, 0 corpo é isolado da pessoa e as experiências subjetivas de saúde e doença são ignorados. No entanto, houve, uma mudança do paradigma mé dico para um conceito mais amplo de comportamento social que se compromete a desenvolver maneiras de medir percepções, sentimentose comportamentos ${ }^{9}$. Como resultado, tem-se passado de uma preocupação com a doença, para uma preocupação com a saúde; não preocupação em curar esim em prevenir e promover saúde; não ênfase aos serviços de saúde e sim ao ambiente social e físico como maiores determinantes de saúde. Logo, o paciente é visto não como um corpo e sim como uma pessoa, dando-seuma crescente importância às experiências subjetivas do indivíduo e às suas interpretações de saúde e doençą ${ }^{9}, 10$. 
Esta mudança de paradigma refletiu-se também na odontologia. Antes, havia uma tendência de tratar cavidade bucal como se fosse uma estrutura anatômica autônoma que acontece de estar localizada em um corpo, mas não está conectado a ele ou a pessoa de maneira alguma. No entanto, com a busca de uma perspectiva mais holística de saúde e doença, esta visão está mudando e isto tem sido acompanhado por duas descobertas: a descoberta de corpo e a descoberta da pessoa, eisto tem resultado em um aumento nas pesquisas preocupadas em ligar as condições bucais a doenças de outras localidades da boca e a conseqüência da saúde na QV ${ }^{9}$.

Locker $^{9}$ relatou que ainda permanece certa confusão a respeito dos conceitos de doença, saúde, SB e QV e as maneiras nas quais estes termos são relacionados. 0 discurso sobre ter SB é confuso e a bocaésemprefoco de análiseao invés de se olhar o indivíduo como um todo, havendo uma ambigüidade do queseria SB esaúde geral e o quanto a SB interfere na QV de um indivíduo. SB é um estado da boca e estruturas associadas onde a doença está contida, a futura doença é inibida, a oclusão é suficiente para mastigar comida e o dente tem uma aparência social saudável $^{11}$. Assim, é uma dentição confortável efuncional que permite $o$ indivíduo a continuar a de sempenhar o papel social ${ }^{12}$.

Sob a ótica da promoção de saúde, a relação entre SB e QV tem sido motivo de atenção dos profissionais da odontologia, principalmente pela relevância de problemas bucais e dos impactos físicos e psicossociais que ela acarreta na vida das pessoas. Os problemas bucais podem causar dor, desconforto, limitações eoutras condições decorrentes de fatores estéticos que afetam a vida social, a alimentação, o exercício de atividades diárias e o bem-estar do indivíduo ${ }^{13}$, acarretando problemas significativos na QV do indivíduo, o que torna essencial entender como o indivíduo percebe a própria condição bucal, pois seu comportamento é condicionado por esta percepção ${ }^{14}$.

Índices como o CPO (cariados, perdidos e obturados) ou o IPC (índice periodontal comunitário) consideram apenas as dimensões clínicas, dependentes do diagnóstico profissional, da cárie dental e das doenças periodontais. Entretanto, alguns problemas possuem fortes raízes sociais e econômicas e só podem ser suficientemente compreen didos e explicados quando seus portadores são ouvidos e quando os autodiagnósticos e opiniões destas pessoas são tomados em consideração. Os indicadores QVRSB deveriam ser vistos não como substitutos para os critérios normativos e sim como um importante complemento a eles 5 .

Qualidade de vida e saúde bucal
em crianças eadolescentes

No caso do indivíduo em desenvolvimento, as propostas para definição de QV apresentadas ainda são muito conflitantes e al gumas características do universo infantil contribuem paraisto. A criança e o adolescente têm diferentes graus de percepção de si mesmos e do mundo, em função da sua fase de desenvolvimento e, com isso, dificilmente podem ser uniformizadosnuma só concepção de satisfação pessoal ${ }^{15}$.

Para crianças e adolescentes, QV pode significar "[...] o quanto seus desejos e esperanças se aproximam do que realmente está acontecendo". Também "reflete sua prospecção, tanto para si, quanto para os outros" e "[...] é muito sujeita a alterações sendo influenciada por eventos cotidianos e problemas crônicos"16. E ainda, segundo Bradlyn et al. ${ }^{17}$, QV podeser definida como multidimensional, pois inclui a interação social, 0 funcionamento físico e emocional da criança e do adolescente e, quando indicado, de sua família, devendo ser um parâmetro sensível às alterações que ocorram no evoluir do desenvolvimento do indivíduo.

Avaliações de QVRSB em pacientes infantis dão uma compreensão das conseqüências das doenças bucais e deformidades na vida da criança; desta maneira, muitas avaliações tradicionais de SB representam uma dimensão unidimensional do aspecto da SB da criança ${ }^{18}$. Isto não significa que os parâmetros clínicos não sejam importantes, eles são com certeza importantes, se não essenciais para mensurar a condição bucal; o problema começa quando os indicadores são comparados com SB e necessidade de tratamento ${ }^{19}$. SB é o padrão de saúde dos tecidos orais e relacionados que permitem um indivíduo comer, falar esocializar sem a atividade da doença, desconforto eembaraço, eo que contribui com o bem-estar geral ${ }^{20}$. Assim, vai além de puramente indicadores clínicos e desta maneira não devem ser igualados com a ausência de doença e deformidade ${ }^{21}$.

\section{Avaliação de qualidade de vida} relacionada à saúde

0 desenvolvimento de instrumentos que avaliem o bem-estar e a QV tem sido cada vez mais 
enfatizado, havendo uma proliferação de instrumentos de avaliação de qualidade de vida relacionada à saúde (QVRS) (Quadro1). Mulhern et al. ${ }^{22}$ propuseram as seguintes características como essenciais a um instrumento de avaliação de QV: (1) incluir a abordagem da função física, desempenho escolar e ocupacional, ajustamento social e auto-satisfação; (2) ter sensibilidade para detectar os problemas funcionais mais comuns de crianças (câncer e aqui pode-se acrescentar "e outras doenças crônicas"); (3) ser confiável e válido para o grupo de pacientes em que será utilizado; (4) ser breve, simples, fácil deadministrar e computar, e reprodutível; (5) valer-se de informação decuidadores familiares ao trato com a criança; (6) ser corrigido para a idade, sob normas populacionais; (7) estar adequado para detectar desempenho acima da média; (8) permitir estimativa confiável do funcionamento pré-mórbido; (9) epermitir à criança capaz de entender 0 conceito de QV ou seus componentes a oportunidade de fornecer sua auto-avaliação.

Quadro 1. Instrumentos de avaliação de qualidade de vida relacionada à saúde bucal.

\begin{tabular}{|c|c|c|c|c|c|c|}
\hline Índice & Autores & Objetivo & Impacto & $\begin{array}{c}\text { Modo de } \\
\text { administração }\end{array}$ & População & $\mathrm{N}^{\circ}$ de itens \\
\hline $\begin{array}{l}\text { Oral Health } \\
\text { Impact } \\
\text { Profile } \\
\text { (OHIP) }\end{array}$ & $\begin{array}{l}\text { Original: } \\
\text { Slade e } \\
\text { Spencer } 44 \\
\text { Formulário } \\
\text { breve: } \\
\text { Slade }\end{array}$ & $\begin{array}{l}\text { Avalia a percepção da } \\
\text { pessoa do impacto social } \\
\text { de alterações orais no } \\
\text { seu bem-estar }\end{array}$ & $\begin{array}{l}\text { Limitação funcional, } \\
\text { desconforto psicológico, } \\
\text { dor física, incapacidade } \\
\text { física, incapacidade } \\
\text { psicológica, } \\
\text { incapacidade e } \\
\text { empecilho social }\end{array}$ & $\begin{array}{l}\text { Auto- } \\
\text { administrado }\end{array}$ & Adultos & $\begin{array}{l}\text { Original: } 49 \\
\text { Formulário } \\
\text { breve: } 14\end{array}$ \\
\hline $\begin{array}{l}\text { The Dental } \\
\text { Impact on } \\
\text { Daily Living } \\
\text { (DIDL) }\end{array}$ & $\begin{array}{l}\text { Leão e } \\
\text { Sheiham }{ }^{46}\end{array}$ & $\begin{array}{l}\text { Comparar } \\
\text { conseqüências } \\
\text { psicossociais } \\
\text { relacionadas à qualidade } \\
\text { de vida com o status em } \\
\text { termos de SB de um } \\
\text { indivíduo ou de uma } \\
\text { comunidade }\end{array}$ & $\begin{array}{l}\text { Aparência, dor, } \\
\text { conforto, performance } \\
\text { geral e restrições } \\
\text { alimentares }\end{array}$ & Questionário & Adultos & 36 \\
\hline $\begin{array}{l}\text { Oral Impacts } \\
\text { on Daily } \\
\text { Performances } \\
\text { (OIDP) }\end{array}$ & $\begin{array}{l}\text { Adulyanon } \\
\text { e Sheiham }{ }^{47}\end{array}$ & $\begin{array}{l}\text { Avaliar o impacto } \\
\text { odontológico de } \\
\text { desempenho diário }\end{array}$ & $\begin{array}{l}\text { Estado da SB, alterações } \\
\text { em tecidos bucais e } \\
\text { satisfação com a } \\
\text { aparência }\end{array}$ & Entrevista & $\begin{array}{l}\text { Adolescentes } \\
\text { e Adultos }\end{array}$ & 8 \\
\hline $\begin{array}{l}\text { Child Oral } \\
\text { Health } \\
\text { Quality of } \\
\text { life } \\
\text { Questionnaire } \\
\text { (COHQoL) }\end{array}$ & $\begin{array}{l}\text { Jokovic et } \\
\text { al. }^{48}\end{array}$ & $\begin{array}{l}\text { Avaliar o impacto físico, } \\
\text { social e psicológico da } \\
\text { saúde bucal na qualidade } \\
\text { de vida }\end{array}$ & $\begin{array}{l}\text { Alterações e doenças } \\
\text { dental, oral e/ou } \\
\text { orofacial }\end{array}$ & $\begin{array}{l}\text { Auto- } \\
\text { administrado }\end{array}$ & Crianças & 49 \\
\hline $\begin{array}{l}\text { Child-Oral } \\
\text { Impact on } \\
\text { Daily } \\
\text { Performance } \\
\text { Index } \\
\text { (CHILD- } \\
\text { OIDP) }\end{array}$ & $\begin{array}{l}\text { Gherunpong } \\
\text { et al. }{ }^{49}\end{array}$ & $\begin{array}{l}\text { Avaliar o impacto } \\
\text { odontológico na } \\
\text { qualidade de vida ea } \\
\text { necessidade de } \\
\text { tratamento } \\
\text { odontológico }\end{array}$ & $\begin{array}{l}\text { Saúde bucal, satisfação } \\
\text { com aparência e } \\
\text { desempenho psicológico } \\
\text { e social }\end{array}$ & $\begin{array}{l}\text { Auto- } \\
\text { administrado }\end{array}$ & Crianças & 8 \\
\hline
\end{tabular}


Embora inúmeros instrumentos sejam utilizados para este fim, ainda não há nenhum instrumento que tenha conseguido abranger todas estas características. Principal mente porquea QV éuma concepção pessoal de difícil quantificação, variando suas definições de acordo com os interesses do indivíduo, de seu grupo cultural e de seus próprios valores. Assim, ela é um conceito global que aborda diferentes facetas da vida de um indivíduo (saúde, família e meio ambiente, entre outras) ${ }^{23}$.

As medidas de QVRSB, mostrando a extensão para qual os distúrbios dentários e bucais condicionam e alteram o desempenho diário físico, psicológico e social, são fundamentais, traduzindo-se em impactos que podem ser categorizados como desempenho funcional efísico (por exemplo, comer e apreciar a comida, e falar e pronunciar com clareza); desempenho psicológico (como sorrir, dar risadas e mostrar os dentes sem constrangimento); e desempenhos sociais (afetando as interações com as pessoas e a realização de tarefas e funções importantes) ${ }^{5}$. Existeuma abundância de mensurações deQVRS desenvolvidos para adultos e que foram utilizados em crianças. Entretanto, mensurações desenvolvidas para adultos podem não ser adequadas para crianças pelo perfil e validade do conteúdo. É imperativo avaliar a confiança, validade e adequação do instrumento para faixa etária em particular do estudo ${ }^{1}$.

Uma estratégia para abordar as diferentes fases de desenvolvimento da criança é criar múltiplas formas de um mesmo instrumento para crianças, cada uma desenhada para diferentes faixas etárias. Esta maneira é vantajosa por levar em consideração os diferentes estágios de desenvolvimento da criança, incluindo sua concepção de doença, assim como sua causa eimpacto. Com esta estratégia, é possível desenhar uma série de questionários garantindo que o formato eo conteúdo sejam apropriados para a idade de todas as crianças ${ }^{24}$.

Tem havido uma crescente preocupação com as populações pediátricas com doenças crônicas epara isso instrumentos demensuração deQVRS genéricos e específicos para doença têm sido desenvolvidos. A avaliação através de um instrumento de QVRS genérico conta com a proteção de populações saudáveis, possibilita comparações padronizadas acerca das condições da saúdecrônica pediátrica efacilita a com paração com populações saudávei $s^{25}$. No entanto, não é capaz de avaliar os sintomas específicos da doença e os efeitos colaterais do tratamento de relevância particular ao grupo doente. Em contraste, avaliações específicas para doenças crônicas (por exemplo, para asma, diabete e artrite) podem prover informações sobre o manejo dessas doenças no nível individual. Todavia, esses instrumentos são incapazes de prover comparação entre grupos saudáveis e doentes. Assim sendo, deve-se integrar uma abordagem combinando os méritos relativos de instrumentos de QVRS genéricos e específicos para doença ${ }^{26}$, a fim de se obter um maior entendimento do impacto da doença nestes pacientes.

Há uma falta de consenso a respeito da administração do instrumento de avaliação de QVRS na criança ou num procurador (por exemplo, responsável ou equipedesaúde). Sempreque a criança for hábil em prover dados confiáveis e válidos, a aplicação direta nestes pacientes é a estratégia ideal porque é consistente com a definição de Q VRS que enfatiza a perspectiva subjetiva do paciente ${ }^{27}$. Entretanto, as crianças, particularmente as pequenas, podem não ser hábeis em prover informação confiável, devendo os pesquisadores avaliar a idade apropriada do instrumento, incluindo o vocabulário, instruções, estrutura das frases, conteúdo e opções de resposta, preocupando-se com as limitações de desenvolvimento. Uma opção para este problema seria desenvolver um instrumento administrado na forma de entrevista que pode incluir procedimentos padronizados para ensinar a criança a responder a mensuração e instruindo-a a não responder questões que não entender, e ainda, checando a compreensão dos itens pela criança. Entretanto, um instrumento administrado por entrevista pode ser mais dispendioso de ser usado que o questionário auto-administrado. Para crianças muito jovens ou gravemente debilitadas, responsáveis podem prover informaçõesque não poderiam ser obtidas de outra forma. Além disso, a perspectiva dos responsáveis é importante por causa da natureza dependente da relação responsável-criança. É esteresponsável quem avalia o impacto da saúde da criança e decide se esta devereceber o tratamento, ele pode também informar o impacto da doença da criança e do tratamento no funcionamento familiar, o que é uma parte integral da QVRS da criança ${ }^{24}$.

Entretanto, existem diversas desvantagens em usar os pais como respondedores. Primeiro, um relato por procuração é de al guma forma inconsistente como conceito de QVRS que é definido de acordo com a opinião subjetiva do paciente ${ }^{24}$. Segundo, o relato de pais e mães pode não ser equival ente entre si ${ }^{28,29}$, sendo recomendado que 
seja feita avaliação dos dois pais e depois cruzar as informações com o objetivo de captar possíveis erros ${ }^{24}$. Terceiro, o relato dos responsáveis sobre o impacto da doença nas suas crianças é baseado no conhecimento acerca de como eles mesmos são afetados. Finalmente, não é totalmente claro que pais sejam os adultos mais adequados a responder o questionário ${ }^{29}$, uma vez que al gumas crianças podem passar mais tempo com professores, cuidadores ou outro membro da família do que com os pais, e assim outro adulto teria um conhecimento maior acerca do funcionamento social e psicológico da criança ${ }^{24}$.

Devido a esta falta de consenso, alguns pesquisadores têm sugerido obter em conjunto informações de responsáveise crian ças ${ }^{28}$. Esta abordagem pode prover uma informação mais completa de como a doença ou o tratamento causam impacto na vida das crianças e suas famílias ${ }^{24}$. No entanto, Kuczynski \& Assumpção ${ }^{15}$ afirmam que é evidentequeainda se está muito aquém de uma concepção uniforme e universal de QV na infância, como também de meios de avaliação deste conceito adaptados ao universo infantil. É prioridade que se tenha clara a necessidade de instituir definições que traduzam os interesses das crianças e do adolescente, e não dos adultos que os avaliam, equeseinstaurem métodos de avaliação que captem a percepção do indivíduo a ser avaliado, e não as expectativas e percepções do cuidador, seja ele pai ou profissional de saúde.

A avaliação da QVRS em criança reflete-sena percepção dos pacientes sobre sua SB e com isso pode melhorar a comunicação entre pacientes, pais e a equipe odontológica ${ }^{30}$. Isto permite um melhor entendimento das conseqüências do estado de SB na vida da criança e da vida da sua família ${ }^{31}$, podendo ainda ajudar na priorização de cuidados e estimar a conseqüência das estratégi as de tratamento e iniciativas ${ }^{32}$.

Qualidade de vida e saú de bucal em pacientes infantis infectados pelo HIV

Desde que a Síndrome da I munodeficiência Adquirida (aids) foi catalogada, o aumento de sua incidência foi progressivo, representando enorme desafio para a medicina. A doença deixou de afetar um grupo específico (homossexuais, hemofílicos e drogados), passando a ocorrer de forma crescente em mulheres e crianças ${ }^{33}$. Graças à melhora do tratamento e evolução dos medicamentos, a infecção pelo vírus da imunodeficiênciahumana (HIV) não émaisuma doença aguda e letal, e tornou-se uma condição crônica e subaguda, embora a ameaça de mortalidade ainda esteja presente. Dessa forma, grande atenção tem sido voltada à QVRS, com as subseqüentes implicações para o longo período de cuidados aos indivíduos HIV-positivos, sua regulação social com a doença e sua interação com os provedores de assistência médica ${ }^{34}$.

Destacam-se, ainda, as condições orais que podem estar afetando a vida dos pacientes pediátricos, uma vez que as manifestações orofaciais são comuns na infecção pelo HIV epodem servir como marcadores da infecção ou prognóstico da progressão do HIV para aids ${ }^{3}$. Dentre as lesões orais encontradas em crianças, destacam-se a candidíase, hipertrofia de parótida, estomatite herpética, leucoplasia pilosa, estomatite aftosa, eritemalinear gengival elinfadenopatia cervica ${ }^{35}$.

A pesar da utilização deterapia medicamentosa em crianças infectadas pelo HIV estar promovendo a diminuição na prevalência de manifestações bucais em tecidos moles ao longo dos anos, observa-se uma constância na al ta prevalência de doença cárie e gengival, sobretudo devido à influência crônica deal guns fatores envolvidos no processo da infecção pelo HIV ${ }^{4}$. Dentre eles, destacam-seo uso prolongado de medicamentosaçucarados ${ }^{4}$, alterações no fluxo salivar pelo uso de medicamentos e/ou por alterações de glândulas salivares ${ }^{36}$, dieta rica em carboidrato ${ }^{4,37}$, repetidos episódios de internação ${ }^{4}$, higiene oral deficiente ${ }^{37}$ eimunossupressão pela infecção pelo HIV ${ }^{37}$.

É importante considerar que nenhuma doença nos tempos atuais fez crescer tanto o número de papéis clínicos, emocionais ou éticos, como fez a infecção pelo HIV ${ }^{38}$. O controle da pandemia de HIV/aids extrapola os limites da intervenção médica ou da saúde pública em sua concepção mais estreita. 0 complexo nosológico HIV/aids assume características de problema social e multifacetado, seja pelas associações doença, sexualidade e morte, seja pelo estigma a ele atribuído ou até mesmo pel as conseqüências econômico-produtivas que acarreta ao infectado ou doente. Assim, não se pode desprezar a força das representações simbólicas do processo saúdedoença e seu impacto sobre a vida dos indivíduos que com ela convivem no seu cotidiano ${ }^{39}$.

A infecção pelo HIV é uma das poucas doenças crônicas e ameaçadoras da vida que afeta tanto pais e criança simultaneamente. É verdadeiramente uma doença familiar onde todos são afe$\operatorname{tados}^{40,41}$. A doença parental pode levar a um aumento dosníveis dedepressão, ansiedadeedificuldades de comportamento da criança ${ }^{42,43}$. Dessa 
forma, a QV do indivíduo portador do vírus HIV é significativamente afetada não só pelas manifestações da infecção, mas também pelo impacto que a doença causa em sua vida. Isto éainda mais relevante ao se considerar os pacientes de baixa idade e ainda em desenvolvimento, uma vez que apresentam doença crônica e podem ter percepção diferenciada quanto a sua QV, quando comparados a outras crianças clinicamente saudáveis.

Por terem de lidar com doença cárie e periodontal, que indubitavelmente podem levar à dor e problemas estéticos, etambém com outras manifestações orais da infecção pelo HIV, torna-se relevante avaliar a QVRS. Todos estes problemas gerados pela doença afetam significativamente 0 cotidiano destas crianças. Seja o desempenho funcional e físico, psicológico e social, todas as esferas sofrem um impacto considerável ao serem analisadas e devem ser levadas em consideração quando se pretende cuidar e tratar estas crianças para que o universo delas seja melhor compreendido e o seu tratamento individualizado.

I dentificar os fatores que reduzem a QV nos casos de HIV/aids é um importante passo para melhorar a QV nesta população, permitindo aos clínicos examinar esses fatores eintervir. Assim, tornam-se cada vez mais necessários instru- mentos que auxiliem o cirurgião-dentista e demais profissionais responsáveis por estas crianças a avaliar não só a presença da enfermidade causada pelo vírus HIV, como também a QVRS destes pacientes infantis, em virtude deste ser um componente auxiliar na mensuração da saúde do paciente.

\section{Conclusão}

A qualidade de vida relacionada à saúde é um importante componente auxiliar aos indicadores clínicos na avaliação da saúde do paciente. Isto se tornaainda mais relevanteem pacientes infantise com doenças crônicas, em que a cura da doença não é possível. Dentre estas doenças crônicas, a infecção pelo HIV merece destaque, uma vez que estes pacientes convivem com os problemas sistêmicos bem como com manifestações bucais da infecção, que determinam um impacto significativo em sua qualidade de vida. Assim, tornam-se cada vez mais necessários instrumentos que auxiliem o cirurgião-dentista e demais profissionais responsáveis por estas crianças para avaliar não só a presença da enfermidade, como também a qualidade de vida destas crianças.

\section{Colaboradores}

AK Buczynski trabalhou na revisão bibliográfica, estruturação e redação do texto. GF Castro e IPR Souza foram orientadoras do estudo, participando da concepção, discussão e revisão final do estudo. 


\section{Referências}

1. M CGrath C, Broder H, Wilson-Genderson M. Assessing the impact of oral health on the quality of children: implications for research and practice. Comm Dent Oral Epid 2004; 32:81-85.

2. Burckhardt CS, Anderson KL. The quality of life scale (QOLS): reliability, validity and utilization. Health and Quality of Life Outcomes 2003; 1:60.

3. Ramos-Gomez FJ, Hilton JF, Canchola AJ, Greenspan D, Greenspan JS, M aldonato YA. Risk factors for HIV-related orofacial soft-tissue manifestations in children. Pediat Dent 1996; 18(2):121-126.

4. Ribeiro AA, Portela M, Souza IP. Relação entre biofilme, atividade de cárie e gengivite em crianças HIV +. Pesqui O dontol Bras 2002; 16(2):144-150.

5. Pinto VG. Saúde bucal coletiva. São Paulo: Editora Santos; 2000.

6. The WHOQOL Group. The development of the World Health Organization quality of life assessment instrument (the WHOQOL). In: Orley J, Kuyken W, editors. Quality of life assessment: international perspectives. Heidelberg: Springer Verlag; 1994.

7. Fleck MPA, Leal OF, Louzada S, Xavier M, Chachamovich E, Vieira G, Pinzon V. Desenvolvimento da versão em português do instrumento de avaliação de qualidade de vida da Organização M undial de Saúde (WHOQOL-100). Rev Bras Psiquiatr 1999; 21(1):19-28.

8. Bastos JM, Saliba NA, Unfer B. Considerações a respeito de SB e classes sociais. Rev Paul Odonto 1996; 38(4): 38-41.

9. Locker D. Concepts of oral health, disease and the quality of life. In: Slade G. M easuring oral health and quality of life. Chapel Hill: University of North Carolina/ Dental Ecology; 1997.

10. M iotto MHMB, Barcellos LA. U ma revisão sobre 0 indicador subjetivo de SB "Oral Health Impact Profile" OHIP. UFES Ver. O dontol. Vitória 2001; 3(1):3233.

11. Yewe-Dwyer M. The definition of oral health. Brit Dent J 1993; 174:224-225.

12. Dolan T. Identification of appropriate outcomes for an aging population. Special Care in Dentistry 1993; 13:35-39.
13. Leão ATT, Cidade MC, Varela JR. Impactos da saúde periodontal na vida diária. Rev Bras Odontol 1998; 5(4):238-241.

14. Barrêto APR, Oliveira CS, Paiva SM, Pordeus IA Qualidade de vida infantil: influência dos hábitos de higiene bucal e do acesso aos serviços odontológicos. Rev Ibero-amer O dontop O dontol Bebê 2004; 7(39):453-460.

15. Kuczynski E, Assumpção JR FP. Definições atuais sobre o conceito de qualidade de vida na infância e adolescência. Ped M od 1999; 35(3):73-78.

16. Hinds P. Quality of life in children and adolescents with cancer. Semin Oncol Nurs 1990; 6:285-291.

17. Bradlyn AS, Ritchey AK, Harris CV, Moore IM, O'Brien RT, Parsons SK, Patterson K, Pollock BH. Quality of life research in Pediatric Oncology. Research Methods and Barriers. Cancer 1996; 78(6):1333-1339.

18. Locker D. Measuring oral health: a conceptual framework. Comm Dent Health 1998; 23:32-36.

19. Sheiham A, Crogg SH. The psychosocial impact of dental diseases on individuals and communities. J Behav Med 1981; 4:257-272.

20. Oral Health Strategy Group. An oral health strategy for England. London: Department of Health; 1994.

21. Cohen LK, Jago JD. Toward the formulation of sociodental indicators. Int J Health Serv 1976; 6:681698.

22. Mulhern RK, Horowitz ME, Ochs J. Assessment of quality of life among Pediatric patients with cancer: Psychological assessment. J Consult Clinic Psychol 1989; 1:130-136.

23. Assumpção JR FB, Kuczynski E, Sprovieri MH, Aranha EM G. Escala de avaliação de qualidade de vida. Arq N europsiquiatria 2000; 58(1): 119-127.

24. Matza LS, Swensen AR, Flood EM, Secnik K, Leidy NK. Assessment of health-related quality of life in children: a review of conceptual, methodological, an regulatory issues. Value in health 2004; 7(1):7992.

25. Varni JW, Burwinkle TM, Seid M, Skarr D. The PedsQ $L^{\text {TM }} 4.00$ as a pediatric population health measure: feasibility, reliability and validity. Ambul Pediatr 2003; 3:329-341. 
26. Varni JW, Burwinkle TM, Lane M M. Health-related quality of life measurement in pediatric clinical practice: an appraisal and percept for future research and application. Health and Quality of Life Outcomes 2005; 3:34.

27. Eiser $C, M$ ohay $H, M$ orse R. The measurement of quality of life in young children. Child: Care, $\mathrm{H}$ ealth and Develop 2000; 26(5):401-414.

28. Eiser C, Morse R. Can parents rate their child's health-related quality of life? Results of a systematic review. Qual Life Res 2001; 10:347-357.

29. Landgraf JM, Abetz LN. M easuring health outcomes in pediatric populations: issue in psychometrics and application. In: Spilker B, editor. Quality of life and pharmacoeconomics in clinical trials. Philadelphia: Lippincott-Raven Publishers; 1996

30. Weintraub JA. Uses of oral health related quality life measures in public health. Comm Dent $\mathrm{H}$ ealth 1998; 15:8-12.

31. Holt RD. Advances in dental public health. Prim Dent Care 2001; 8:99-102

32. Sheiham A, M aizels JE, Cushing AM. The concept of need in dental care. Int Dent J 1982; 32:265-270.

33. Valentim C. Condições patológicas da cavidade bucal na infância. In: Corrêa M SN P. O dontopediatria na primeira infância. São Paulo: Editora Santos; 1998.

34. Elliott AJ, Russo J, Roy-Byrne PP. The effect of changes in depression on health related quality of life (HRQ oL) in HIV infection. Gen Hosp Psychiatry 2002; 24:43-47.

35. Chigurupati R, Raghavan SS, Studen-Pavlovich DA. Pediatric infection and its manifestations: a review. Pediatric Dent 1996; 18(2):106-113.

36. Ribeiro AA, Bunzman ER, Castro GF, Nishio $C$, Souza IP. Relation between salivary flow, medicines and caries in HIV + children. J D ent Res 1998; 77:841.

37. Souza IPR, Teles GS, Castro GF, Guimarães L, Viana RBC, Peres $M$. Prevalência de cárie em crianças infectadas pelo HIV. Rev Bras Odontol 1996; 53(1):49-51.

38. Sonis ST, Fazio RC, Fang L. Princípios e prática de medicina oral. $2^{\underline{a}}$ ed. Rio de Janeiro: Guanabara Koogan; 1996.
39. Santos EM, Carvalho AMT. Crônicas da vida mais contrariada: sofrimento psíquico, HIV/AIDS e trabalho em saúde. Cad Saúde Col 1999; 7(2):147-173

40. Gibb DM, Duggan C, Lwin R. The family and HIV. Genitourinary Medicine 1991; 67:363-366.

41. Melvin $D$, Sherr $L$. The child in the family - Responding to AIDS and HIV. AIDS Care 1993; 5(1):3542.

42. Anderson CA, Hamen CL. Psychosocial outcomes of children of unipolar depressed, bipolar, medically ill and normal women: a longitudinal study. J Consult Clinic Psychol 1993; 61:448-454.

43. Siegal K, M esagno FP, Karus D, Christ G, Banks K, Moynihan R. Psychosocial adjustment of children with terminally ill parent. J American Academy Child Adolescent Psychiatry 1992; 31(2):327-333.

44. Slade GD, Spencer AJ. Development and evolution of the oral health impact profile. Com Dent $\mathrm{Health}$ 1994; 11:3-11.

45. Slade GD. Derivation and validation of a short form oral health impact profile. Com Dent Oral Epid 1997; 25:284-290.

46. Leão A, Sheiham A. The development of measures on daily impacts on daily living. Comm Dental Health 1996; 13:22-26.

47. Adulyanon S, Sheiham A. Oral impacts on Daily performances. In: Slade GD. M easuring Oral health and Quality of Life. Chapel Hill: University of N orth Carolina; 1997.

48. Jokovic A, Locker D, Stephens M, Kenny D, Tompson B, Guyatt G. Validity and reliability of a questionnaire for measuring child oral-health-related quality of life. J Dent Res 2002; 81:459-463.

49. Gherunpong S, Tsakos G, Sheiham A. Developing and evaluating an oral heath-related quality of life, impact for children; the OIDP-CHILD. Com Dent $\mathrm{H}$ ealth 2004; 21(2):161-169.

Artigo apresentado em 30/05/2006

Aprovado em 22/05/2007

Versão final apresentada em 15/06/2007 